\title{
The Influence of Birth Quartile, Maturation, Anthropometry and Physical Performances on Player Retention: Observations from an Elite Football Academy
}

Rickesh Patel, ${ }^{1}$ Alan Nevill, ${ }^{1}$ Tina Smith, ${ }^{1}$ Ross Cloak, ${ }^{1}$ Matthew Wyon ${ }^{1}$

Abstract

Understanding the factors that influence player retention within elite youth football can be used to enhance current practices. This study investigated an English Category 1 academy to determine if birth quartile, somatic maturity, anthropometric and physical performance characteristics are associated with player retention across the developmental pathway. Birth dates of 355 elite players from U11 to U21 groups were categorised into birth quartiles and logistic regression (odds ratio) analysis was used to determine differences in retention. Multilevel modelling compared somatic maturity, anthropometry, countermovement jump, sprint time (10 and $30 \mathrm{~m})$, agility T-test, Yo-Yo Intermittent Recovery Level 1 or 2 performance between retained and dropout players. Logistic regression (odds ratio) analysis revealed no significant differences between birth quartiles for the likelihood of being retained across age groups. Multilevel modelling revealed that retained players were typically older, advanced in maturity, and superior in body size and physical performances compared to dropouts, with small to medium effect sizes typically observed. This study indicates that within a highly selective cohort of young football players, somatic maturity, anthropometric and physical performance characteristics, but not birth quartile, distinguish individuals that are subsequently retained or dropout in an age group dependent manner. Youth football organisations should seek to implement multidisciplinary and dynamic talent selection and retention strategies in order to prevent the inappropriate discrimination and loss of talented young players.

Key words

Soccer, talent selection, relative age effect, biological maturation, fitness 


\section{Introduction}

Youth football development programmes operated by professional clubs principally aim to progress talented young players into the respective senior team ${ }^{1}$. In England, players can be formally recruited from 9 years of age by academies in order to develop a range of competencies deemed necessary for football performance ${ }^{2}$, within an elite-level environment which includes high quality facilities and coaching ${ }^{1}$. Previous research demonstrates that individuals selected by academy or elite-level youth teams can be distinguished from lower-level peers through a multitude of factors. Specifically, the former exhibit superior sport-specific ${ }^{3-5}$, perceptual-cognitive and psychological skills ${ }^{6-}$ 8 , anthropometric and/or physical performance characteristics ${ }^{3-5,7,8}$, as well as advanced chronological age and/or biological maturity ${ }^{3,8}$. Of these factors, the most extensively studied in relation to talent identification in male football are birth date, biological maturation, anthropometry and physical performances ${ }^{9}$. In order for talent identification and selection processes to be highly successful, it is important to appraise current practices so that suitable improvements can be made in light of contemporary research.

The relative age effect is a well-established selection bias across youth sports, with players born towards the start of the selection year highly represented within elite-level youth teams compared to relatively younger players ${ }^{10,11}$. In addition, another selection bias relates to the preferential selection of players that are on-time or advanced in biological maturity ${ }^{12,13}$. Whilst there is a clear relative age effect observed in academy teams, anthropometrical and physical performance characteristics are typically similar between birth quartiles, due to the variability of growth and maturation processes ${ }^{14,15}$, though other age-related differences may be apparent between the oldest and youngest players within a cohort ${ }^{16}$. Therefore, selection biases due to relative age and/or biological maturation appear related to the observation that these factors are predictors of anthropometry and physical performances ${ }^{17}$.

In light of these selection biases, many talented young players that are born towards the end of the selection year and/or later maturing have been shown to prematurely dropout of football ${ }^{18,19}$. This loss of talent at a young age would appear to contravene one of the main outcomes of football academies, that is, to develop talented youth into professional players ${ }^{1}$. However, albeit limited in number, recent evidence from across Europe suggests that the small number of relatively younger and/or later maturing players that are selected into elite teams have a greater prospect of long-term success defined as attaining professional status ${ }^{20,21}$. This relates to observations of an 'underdog' hypothesis, whereby developmental advantages are gained through playing with older (and earlier maturing) peers ${ }^{22}$.

The aforementioned highlight problematic aspects of player recruitment in football academies. First, it is clear that youth teams discriminate against the selection of relatively younger players, yet, the extent to which birth date subsequently influences 
the likelihood of being retained throughout the developmental pathway remains unclear. Second, whilst maturation, anthropometry and physical performance characteristics are able to distinguish between elite and non-elite youth players, the suitability of these factors to determine the most successful players within highly selective cohorts appears questionable ${ }^{23,24}$.

To date, few studies have compared the characteristics of players that either persist or dropout from football academies. Deprez et al. identified that in 8 to 16 year old players selected by elite youth teams in Belgium, physical performances, but not age, anthropometry or maturation were able to distinguish retained and dropout players from each annual age group (U10 to U17) ${ }^{25}$. However, it remains unclear if physical performances and anthropometry, as opposed to other relevant factors, exert an influence on the retention process beyond these ages, and ultimately towards attaining professional status. Furthermore, as talent identification and selection policies may differ across nations, due to multiple factors including task and environmental constraints as well as socio-cultural influences ${ }^{9}$, the characteristics that distinguish retained and dropout players may also vary.

The purpose of this study was to perform a longitudinal investigation of factors associated with retention across a broad age range within an English football academy. To the authors awareness, this is the first study to explore the retention process within an English Category 1 Academy (i.e. top categorised) across the developmental pathway, including entry to the First Team squad (i.e. retention from the U21 group). The primary aim was to investigate the influence of birth quartile on the likelihood of being retained between U11 and U21 age groups. The secondary aim was to investigate the differences in somatic maturity, anthropometric and physical performance characteristics between retained and dropout players from each age group between U11 and U21.

\section{Methods}

Aim one sought to investigate the influence of birth quartile on retention; 355 individual male football players from one English professional club with Category 1 Academy status were included. Players were born between 1990 and 2006 and were registered to the club between 2010/11 and 2016/17 seasons. Players represented an age group for each season they were registered to the club (i.e. U11 through U16, U18, U21).

Aim two sought to investigate differences between players that were identified as retained or dropout, which included 3016 data points from 353 players born between 1990 and 2006, from the same football club. All players performed physical testing as part of normal procedures between $2010 / 11$ to 2016/17 seasons. Players represented an age group for each season they were registered to the club (i.e. U11 through U16, U18, U21). Data was collected on up to four testing periods per season, separated by approximately three months, for a total of seven seasons. Accordingly, players were 
followed for each season they were registered to the club and typically had repeated measurements (up to a maximum of four) for each season they were retained. All available individual player data was included, corresponding to mixed-longitudinal data. The total number of measurements obtained for individual players were as follows: 1 $(n=14), 2(n=28), 3(n=46), 4(n=23), 5(n=23), 6(n=31), 7(n=23), 8(n=21), 9(n=26), 10$ $(n=20), 11(n=11), 12(n=11), 13(n=13), 14(n=9), 15(n=9), 16(n=4), 17(n=6), 18(n=7)$, $19(n=0), 20(n=3), 21(n=2), 22(n=5), 23(n=3), 24(n=2), 25(n=2), 26(n=1), 27(n=8), 28$ $(n=2)$.

For both aims, each player was assigned to either a "retained" or "dropout" group according to their playing status throughout the study. Retained players were individuals that remained registered to the club in the following season they completed testing for a respective age group, and thus represented the subsequent age group in the following season. Dropout players were individuals that were no longer affiliated with the club in the following season after they were tested in a respective age group.

An observational design was used to investigate birth quartile, maturation, anthropometry and physical performance characteristics of male academy football players identified as retained or dropout. All players were grouped into cohorts based on their chronological age, with the selection year spanning from September in one year to August of the following year. Players were only included in this study if they would qualify for home-grown status according to the Premier League. Specifically, players were required to be registered to this club or any other club (prior to being recruited by this club) affiliated with The Football Association or Football Association Wales for three seasons or 36 months prior to their $21^{\text {st }}$ birthday ${ }^{1}$. All players at the club completed routine monitoring and provided signed consent as part of contractual agreements with the club to allow their anonymised data to be used for research purposes. This study received ethical approval from the ethics committee at the University of Wolverhampton and permission from the club to use the anonymised data.

\section{Procedures}

All players completed standardised physical tests throughout the season, conducted by a team of trained exercise scientists that were employed by the club (as sports scientists) and The Premier League (as fitness testing assistants). All testing was completed indoors within a gymnasium and on an artificial turf pitch. At each testing session, all tests were carried out in the same sequence according to previously outlined recommendations by the Premier League ${ }^{1}$. Specifically, this involved: anthropometry, standardised jump-based warm up, jump test, standardised running-based warm up, sprint and agility tests, followed by Yo-Yo Intermittent Recovery Level 1 or 2 . The jump and running-based warm ups were standardised and consisted of dynamic movements in the gymnasium and on the artificial pitch, respectively, for $10 \mathrm{~min}$ each. Players had a minimum of 5 min recovery between tests, at least 60 s passive recovery between attempts for sprint and agility tests, and up to $20 \mathrm{~s}$ between attempts for the jump test. 
Players performed all tests in football boots, apart from the jump test which were performed in running shoes. All players were familiarised with testing procedures and were provided with strong verbal encouragement throughout.

\section{Anthropometry}

All anthropometric measures were obtained according to The International Society for the Advancement of Kinanthropometry (ISAK) protocols ${ }^{26}$ by a team of ISAK accredited exercise scientists. Anthropometric testing was performed in the morning prior to training and in a t-shirt and shorts only. Standing height was assessed using a stadiometer (Model HR001, Tanita Leicester Height Measure) to the nearest $0.1 \mathrm{~cm}$. Sitting height was assessed using an anthropometric box (height of $40 \mathrm{~cm}$ ), as per ISAK guidelines ${ }^{26}$, positioned at the base of the stadiometer, with measurement recorded to the nearest $0.1 \mathrm{~cm}$. Leg length was calculated as the difference between standing height and sitting height. Body mass (Seca 22089, Hamburg, Germany) was assessed to the nearest $0.1 \mathrm{~kg}$.

\section{Physical performance}

Jump performance was assessed using the countermovement jump (OptoJump, Microgate, Bolzano, Italy) test to the nearest $0.1 \mathrm{~cm}$. The countermovement jump (CMJ) was performed with players starting in an upright position, rapidly going into a squat position with knees flexed at approximately 90 degrees, thereafter jumping maximally and landing with minimal knee flexion. Hands remained on the hips to negate the influence of arm swing. The highest of three jump trials was recorded.

Sprint performance was assessed through three maximal sprints of $30 \mathrm{~m}$ measured to the nearest $0.01 \mathrm{~s}$ using timing gates (Brower Timing System, Utah, USA). Players commenced each sprint from a standing start with their front foot $0.5 \mathrm{~m}$ behind the first timing gate. The players began when ready, thus nullifying the influence of reaction time. The fastest $10 \mathrm{~m}$ split time and $30 \mathrm{~m}$ time were recorded, which could have occurred in different trials.

Agility performance was assessed using a modified version of the agility T-test ${ }^{27}$. Players commenced each sprint from a standing start with their front foot $0.5 \mathrm{~m}$ behind the timing gate. Subsequently, players ran forward $10 \mathrm{~m}$, turned right 90 degrees around a cone and ran forward $5 \mathrm{~m}$, turned right 180 degrees around another cone and ran forward $10 \mathrm{~m}$, turned right 180 degrees and ran forward $5 \mathrm{~m}$, turned right 90 degrees and ran $10 \mathrm{~m}$ to the start/finishing line. Players began when ready, thus nullifying the influence of reaction time. The fastest recorded time of three attempts to the left and right as well as the composite score determined using the fastest time from each direction were recorded to the nearest $0.01 \mathrm{~s}$.

Aerobic capacity was determined via the Yo-Yo Intermittent Recovery Level 1 (Yo-Yo IR1) for U11 to U15 players and Level 2 (Yo-Yo IR2) for U16 to First Team players ${ }^{28,} 29$. Players performed $2 \times 20$ m shuttles with a progressively increasing speed controlled by 
an audio recording. Players had $10 \mathrm{~s}$ active rest between each 20-m shuttle run, which involved walking $2 \times 5 \mathrm{~m}$. Players ran until they failed to reach the finishing line on two occasions, with the final score recorded as the distance of the last successfully completed shuttle.

\section{Relative Age}

Players birthdates were obtained from club records and categorised into birth quartiles (Q) within each age group according to the selection year spanning $1^{\text {st }}$ September to $31^{\text {st }}$ August; Q1= September-November, Q2=December-February, Q3=March-May, Q4=JuneAugust.

\section{Maturity status}

Estimation of biological maturity was calculated for players in U11 to U16 groups only, using Equation 3 of the non-invasive method developed by Mirwald et al., which estimates maturity offset within an error of \pm 1 year $95 \%$ of the time ${ }^{30}$. Age at peak height velocity (APHV) was subsequently calculated from chronological age and maturity offset which was updated at each testing session, where weight in equation 3 represents body mass reported in this study.

\section{Statistical analysis}

To investigate the likelihood of being retained (dependent variable) from each age group across birth quartiles (independent variable), a binary logistic regression was conducted to derive odds ratios (OR) with 95\% confidence intervals $(\mathrm{Cl})$ for betweenquartile comparisons, with Q1 as the referent group. Logistic regression (odds ratio) analysis was conducted using SPSS version 24 , with statistical significance accepted at the $95 \%$ confidence level $(P<0.05)$.

To investigate differences in somatic maturity, anthropometric and physical performance characteristics (dependent variables) between retained and dropout players (independent variable) in each age group (U11 through U21), mixed-longitudinal data was analysed using multilevel modelling (MLwiN software package, v 3.02, Bristol University, Bristol, UK). Multilevel modelling is an extension of ordinary multiple regression where the data have a hierarchical or clustered structure. A hierarchy consist of units or measurements grouped at different levels. In our example, individual players are the level 2 variation and each of their repeated measurements are the nested observations at level 1 . Here, the multilevel modelling software assumes the players to be a random sample (of academy footballers throughout the UK) that represent the level 2 units, with players' repeated measurements recorded from each visit being nested below as level 1 units. To the authors' awareness, this is the first time multilevel modelling has been used to address this research question. Multilevel modelling was deemed the most appropriate method for analysis of this dataset ${ }^{31}$, particularly as the same number of measurement occasions per player is not necessary ${ }^{32}$. Data was initially split according to standard age groups within the club. Thereafter, a model for 
each age group and dependent variable (chronological age, somatic maturity [U11-U16 only], height, body mass, CMJ, agility composite, $10 \mathrm{~m}$ sprint time, $30 \mathrm{~m}$ sprint time and Yo-Yo Intermittent Recovery Level 1 or 2) was created separately, allowing for each player to be our level 2 variation (between-subject) and repeated measurements for each player to be our level 1 variation (within-subject). Furthermore, Cohen's $d$ effect sizes (ES) and thresholds ( $0.2,0.5$ and 0.8 corresponding to small, medium and large, respectively) ${ }^{33}$ were used to compare the magnitude of differences between retained and dropout players for each dependent variable. Statistical significance was accepted at the $95 \%$ confidence level $(P<0.05)$. The following model was used to determine differences between retained and dropout players for dependent variables within each age group:

$y_{i j}=\beta_{0}+\beta_{1} x_{j}+u_{j}+e_{i j}$

where $y$ is the dependent variable of interest on measurement occasion $i$ for the $j$ th player, and $x_{j}$ corresponds to their playing status (i.e. a $[0,1]$ indicator variable where 0 is for retained, and 1 is for dropout); the parameter $\beta_{0}$ is called the intercept and corresponds to the overall mean of the dependent variable for retained players (our baseline or reference group); the parameter $\beta_{1}$ represents the difference between the retained and dropout players, specifically in the mean of the dependent variable. The symbol $u_{j}$ represents the between-subjects (level 2 units) variable (assumed Normal (0, $\left.\sigma_{u}^{2}\right)$ ) and the symbol $e_{i j}$ represents the within-subjects (level 1 units) variable (assumed Normal $\left.\left(0, \sigma_{e}^{2}\right)\right)$, where both terms are assumed to be independent.

\section{Results}

\section{Birth Quartile Analysis:}

Examination of player retention from each age group (Table 1) revealed that Q4 players had the highest proportion of dropout in the U11 group; thereafter, the highest dropout from each age group came from Q1 or Q3. Players from Q1 contributed around half of the absolute number of dropout from each age group, where this was higher in U12 and U13 groups. Odds ratios revealed only one significant difference, with Q3 players in the U21 group 4.0 times more likely to be retained in comparison with Q1 (95\% Cl: 1.115.5). Players from $Q 4$ tended to have a greater likelihood of being retained compared with Q1, and this was particularly evident in U13, U16 and U21 groups. 
Table 1 Number (\%) of elite youth players from each birth quartile identified as dropout or retained from each age group within the club, as well as quartile (Q) comparisons for the likelihood of being retained.

\begin{tabular}{|c|c|c|c|c|c|c|c|c|c|}
\hline & & \multicolumn{4}{|c|}{ Birth quartile } & \multirow[b]{2}{*}{ Total } & \multirow{2}{*}{$\begin{array}{l}\text { Q2 vs Q1 OR } \\
(95 \% \mathrm{Cl})\end{array}$} & \multirow{2}{*}{$\begin{array}{l}\text { Q3 vs Q1 OR } \\
(95 \% \mathrm{Cl})\end{array}$} & \multirow{2}{*}{$\begin{array}{l}\text { Q4 vs Q1 OR } \\
(95 \% \mathrm{Cl})\end{array}$} \\
\hline & & Q1 & Q2 & Q3 & Q4 & & & & \\
\hline \multirow[t]{2}{*}{ U11 } & Dropout & $8(14.8 \%)$ & $4(12.9 \%)$ & $3(15.8 \%)$ & $3(30.0 \%)$ & $18(15.8 \%)$ & $1.2(0.32-4.3)$ & $0.9(0.2-3.9)$ & $0.4(0.1-1.9)$ \\
\hline & Retained & $46(85.2 \%)$ & 27 (87.1\%) & $16(84.2 \%)$ & 7 (70.0\%) & $96(84.2 \%)$ & & & \\
\hline \multirow[t]{2}{*}{ U12 } & Dropout & $8(14.5 \%)$ & 4 (9.8\%) & 1 (6.3\%) & $1(14.3 \%)$ & 14 (11.8\%) & $1.6(0.4-5.6)$ & $2.6(0.3-22.1)$ & $1.0(0.1-9.6)$ \\
\hline & Retained & 47 (85.5\%) & 37 (90.2\%) & 15 (93.8\%) & $6(85.7 \%)$ & 105 (88.2\%) & & & \\
\hline \multirow[t]{2}{*}{ U13 } & Dropout & $9(16.7 \%)$ & 2 (4.4\%) & $0(0.0 \%)$ & $0(0.0 \%)$ & 11 (8.7\%) & $4.3(0.9-21.0)$ & $\wedge$ & $\hat{n}$ \\
\hline & Retained & 45 (83.3\%) & $43(95.6 \%)$ & $21(100 \%)$ & $6(100 \%)$ & 115 (91.3\%) & & & \\
\hline \multirow[t]{2}{*}{ U14 } & Dropout & 20 (35.1\%) & 15 (31.3\%) & 9 (37.5\%) & $2(28.6 \%)$ & 46 (33.8\%) & $1.2(0.5-2.7)$ & $0.9(0.3-2.4)$ & $1.4(0.2-7.6)$ \\
\hline & Retained & 37 (64.9\%) & $33(68.8 \%)$ & $15(62.5 \%)$ & 5 (71.4\%) & $90(66.2 \%)$ & & & \\
\hline \multirow[t]{2}{*}{ U15 } & Dropout & $11(22.0 \%)$ & $6(15.8 \%)$ & $3(15.0 \%)$ & $1(16.7 \%)$ & $21(18.4 \%)$ & $1.5(0.5-4.5)$ & $1.6(0.4-6.5)$ & $1.4(0.1-13.4)$ \\
\hline & Retained & 39 (78.0\%) & $32(84.2 \%)$ & $17(85.0 \%)$ & 5 (83.3\%) & $93(81.6 \%)$ & & & \\
\hline \multirow[t]{2}{*}{ U16 } & Dropout & $20(45.5 \%)$ & $19(48.7 \%)$ & $10(50.0 \%)$ & $1(16.7 \%)$ & $50(45.9 \%)$ & $0.8(0.4-2.1)$ & $0.8(0.3-2.4)$ & $4.2(0.4-38.7)$ \\
\hline & Retained & $24(54.5 \%)$ & 20 (51.3\%) & $10(50.0 \%)$ & 5 (83.3\%) & 59 (54.1\%) & & & \\
\hline \multirow[t]{2}{*}{ U18 } & Dropout & $12(34.3 \%)$ & 7 (21.9\%) & $9(52.9 \%)$ & 2 (40.0\%) & 30 (33.7\%) & $1.9(0.6-5.5)$ & $0.5(0.1-1.5)$ & $0.8(0.1-5.3)$ \\
\hline & Retained & $23(65.7 \%)$ & $25(78.1 \%)$ & $8(47.1 \%)$ & $3(60.0 \%)$ & $59(66.3 \%)$ & & & \\
\hline \multirow[t]{2}{*}{ U21 } & Dropout & 17 (73.9\%) & 14 (51.9\%) & $7(41.2 \%)$ & $1(25.0 \%)$ & 39 (54.9\%) & $2.6(0.8-8.7)$ & $4.0(1.1-15.5)^{\#}$ & $8.5(0.7-98.2)$ \\
\hline & Retained & $6(26.1 \%)$ & $13(48.1 \%)$ & $10(58.8 \%)$ & $3(75.0 \%)$ & 32 (45.1\%) & & & \\
\hline
\end{tabular}

Notes: $\mathrm{OR}=$ odds ratio; $95 \% \mathrm{Cl}=95 \%$ confidence intervals

\# denotes significant difference vs Q1 $(\mathrm{P}<0.05)$. ^denotes calculation could not be completed due 


\section{Maturation, Anthropometry and Physical Performances:}

Several statistically significant differences between retained and dropout players were observed in an age group dependent manner, with retained players typically older, advanced in somatic maturity, taller, heavier, and superior in physical performances (see Table 2). Retained players in the U12 group were significantly older and faster in the agility T-test and $30 \mathrm{~m}$ sprint. A significantly lower APHV was observed in the U13 group, along with superior agility T-test performance. Players retained in the U14 group were significantly older, taller, heavier, and faster in the agility T-test as well as $10 \mathrm{~m}$ and $30 \mathrm{~m}$ sprint tests. In the U15 group, retained players were significantly faster in the agility T-test. Retained players in the U16 group were significantly older and faster in the $10 \mathrm{~m}$ sprint test. Retained players in the U18 group achieved a significantly faster $30 \mathrm{~m}$ sprint time. In the U21 group, retained players were significantly faster in the agility Ttest. 
Table 2 Somatic maturity, anthropometric and physical performance characteristics of elite youth players identified as retained or dropout from U11 to U21 groups between 2010/11 and 2016/17 seasons.

\begin{tabular}{|c|c|c|c|c|c|c|c|c|c|c|c|c|c|}
\hline \multirow{2}{*}{$\begin{array}{l}\text { Age } \\
\text { Group }\end{array}$} & \multirow{2}{*}{ Variable } & \multicolumn{3}{|c|}{ Retained } & \multicolumn{3}{|c|}{ Dropout } & \multirow{2}{*}{ ES } & \multirow{2}{*}{ Interpretation } & \multicolumn{2}{|c|}{$\begin{array}{c}\text { Between-Subject } \\
\text { Variance }\end{array}$} & \multicolumn{2}{|c|}{$\begin{array}{c}\text { Within-Subject } \\
\text { Variance }\end{array}$} \\
\hline & & $\mathbf{n}$ & Mean & SE & $\mathbf{n}$ & Mean & SE & & & Mean & SE & Mean & SE \\
\hline \multirow[t]{9}{*}{ U11 } & CA (y) & 96 & 11.0 & 0.0 & 17 & 10.9 & 0.1 & -0.39 & Small & $0.1^{\wedge}$ & 0.0 & $0.1^{\wedge}$ & 0.0 \\
\hline & APHV (y) & 94 & 13.3 & 0.0 & 15 & 13.3 & 0.1 & 0.07 & Small & $0.1^{\wedge}$ & 0.0 & $0.0^{\wedge}$ & 0.0 \\
\hline & Height (cm) & 94 & 147.7 & 0.7 & 15 & 145.1 & 1.8 & -0.32 & Small & $37.9^{\wedge}$ & 5.4 & $4.5^{\wedge}$ & 0.5 \\
\hline & Body Mass (kg) & 94 & 38.5 & 0.5 & 15 & 36.1 & 1.3 & -0.40 & Small & $20.5^{\wedge}$ & 3.0 & $4.5^{\wedge}$ & 0.5 \\
\hline & $\mathrm{CMJ}(\mathrm{cm})$ & 92 & 27.0 & 0.4 & 14 & 25.0 & 1.2 & -0.38 & Small & $13.9^{\wedge}$ & 2.3 & $8.2^{\wedge}$ & 0.8 \\
\hline & Agility Comp. (s) & 94 & 20.89 & 0.09 & 14 & 21.40 & 0.26 & 0.45 & Medium & $0.54^{\wedge}$ & 0.11 & $0.61^{\wedge}$ & 0.06 \\
\hline & 10 m Sprint (s) & 93 & 1.96 & 0.01 & 14 & 2.01 & 0.03 & 0.46 & Medium & $0.01^{\wedge}$ & 0.00 & $0.00^{\wedge}$ & 0.00 \\
\hline & 30 m Sprint (s) & 94 & 5.04 & 0.03 & 14 & 5.13 & 0.08 & 0.28 & Small & $0.06^{\wedge}$ & 0.01 & $0.02^{\wedge}$ & 0.00 \\
\hline & Yo-Yo IR1 (m) & 41 & 885 & 45 & 6 & 785 & 142 & -0.27 & Small & $43817 \wedge$ & 18509 & 11504 & 17055 \\
\hline \multirow[t]{9}{*}{ U12 } & CA (y) & 106 & $12.0^{*}$ & 0.0 & 13 & 11.8 & 0.1 & -0.53 & Medium & $0.1^{\wedge}$ & 0.0 & $0.1^{\wedge}$ & 0.0 \\
\hline & APHV (y) & 103 & 13.6 & 0.0 & 13 & 13.5 & 0.1 & -0.25 & Small & $0.2^{\wedge}$ & 0.0 & $0.0^{\wedge}$ & 0.0 \\
\hline & Height (cm) & 103 & 154.1 & 0.7 & 13 & 151.5 & 2.0 & -0.30 & Small & $44.3^{\wedge}$ & 6.1 & $5.0^{\wedge}$ & 0.5 \\
\hline & Body Mass (kg) & 103 & 42.6 & 0.6 & 13 & 43.5 & 1.9 & 0.11 & Small & $38.6^{\wedge}$ & 5.2 & $3.0^{\wedge}$ & 0.3 \\
\hline & $\mathrm{CMJ}(\mathrm{cm})$ & 105 & 27.5 & 0.5 & 12 & 25.9 & 1.4 & -0.28 & Small & $17.5^{\wedge}$ & 2.8 & $9.7 \wedge$ & 1.0 \\
\hline & Agility Comp. (s) & 106 & $20.73^{*}$ & 0.09 & 12 & 21.40 & 0.28 & 0.61 & Medium & $0.56^{\wedge}$ & 0.10 & $0.58 \wedge$ & 0.06 \\
\hline & 10 m Sprint (s) & 104 & 1.95 & 0.01 & 12 & 2.01 & 0.03 & 0.47 & Medium & $0.01^{\wedge}$ & 0.00 & $0.00^{\wedge}$ & 0.00 \\
\hline & 30 m Sprint (s) & 105 & $4.98 *$ & 0.03 & 12 & 5.18 & 0.08 & 0.59 & Medium & $0.06^{\wedge}$ & 0.01 & $0.03^{\wedge}$ & 0.00 \\
\hline & Yo-Yo IR1 (m) & 44 & 982 & 48 & 5 & 574 & 164 & -1.07 & Large & $63382^{\wedge}$ & 21867 & $96249 \wedge$ & 15690 \\
\hline \multirow[t]{5}{*}{ U13 } & CA (y) & 115 & 13.0 & 0.0 & 8 & 13.1 & 0.1 & 0.42 & Small & $0.0^{\wedge}$ & 0.0 & $0.1^{\wedge}$ & 0.0 \\
\hline & APHV (y) & 113 & $13.6 *$ & 0.0 & 8 & 14.0 & 0.2 & 0.58 & Medium & $0.2^{\wedge}$ & 0.0 & $0.0^{\wedge}$ & 0.0 \\
\hline & Height (cm) & 113 & 161.6 & 0.7 & 8 & 157.0 & 2.9 & -0.46 & Medium & $58.7^{\wedge}$ & 7.8 & $5.4^{\wedge}$ & 0.5 \\
\hline & Body Mass (kg) & 113 & 49.1 & 0.7 & 8 & 44.0 & 2.6 & -0.55 & Medium & $49.9^{\wedge}$ & 6.6 & $4.2^{\wedge}$ & 0.4 \\
\hline & $\mathrm{CMJ}(\mathrm{cm})$ & 112 & 30.8 & 0.5 & 8 & 27.2 & 1.9 & -0.60 & Medium & $22.9 \wedge$ & 3.4 & $7.2^{\wedge}$ & 0.7 \\
\hline
\end{tabular}




\begin{tabular}{|c|c|c|c|c|c|c|c|c|c|c|c|c|c|}
\hline & Agility Comp. (s) & 112 & $20.23 *$ & 0.07 & 8 & 20.85 & 0.28 & 0.67 & Medium & $0.48^{\wedge}$ & 0.07 & $0.21^{\wedge}$ & 0.02 \\
\hline & 10 m Sprint (s) & 111 & 1.88 & 0.01 & 8 & 1.94 & 0.03 & 0.53 & Medium & $0.01^{\wedge}$ & 0.00 & $0.01^{\wedge}$ & 0.00 \\
\hline & 30 m Sprint (s) & 111 & 4.79 & 0.02 & 8 & 4.96 & 0.09 & 0.54 & Medium & $0.05^{\wedge}$ & 0.01 & $0.02^{\wedge}$ & 0.00 \\
\hline & Yo-Yo IR1 (m) & 45 & 1109 & 46 & 6 & 874 & 120 & -0.68 & Medium & $45718^{\wedge}$ & 19659 & $98414 \wedge$ & 17220 \\
\hline \multirow[t]{9}{*}{ U14 } & CA (y) & 93 & $14.0^{*}$ & 0.0 & 42 & 13.8 & 0.1 & -0.36 & Small & $0.0^{\wedge}$ & 0.0 & $0.1^{\wedge}$ & 0.0 \\
\hline & APHV (y) & 93 & 13.5 & 0.1 & 40 & 13.6 & 0.1 & 0.16 & Small & $0.3^{\wedge}$ & 0.0 & $0.0^{\wedge}$ & 0.0 \\
\hline & Height (cm) & 93 & $170.0^{*}$ & 0.8 & 40 & 167.0 & 1.4 & -0.28 & Small & $53.9^{\wedge}$ & 6.9 & $5.5^{\wedge}$ & 0.5 \\
\hline & Body Mass (kg) & 93 & $57.4^{*}$ & 0.8 & 40 & 53.9 & 1.5 & -0.31 & Small & $60.0^{\wedge}$ & 7.6 & $5.1^{\wedge}$ & 0.5 \\
\hline & $\mathrm{CMJ}(\mathrm{cm})$ & 89 & 34.2 & 0.6 & 36 & 32.6 & 1.1 & -0.22 & Small & $26.2^{\wedge}$ & 3.8 & $7.7 \wedge$ & 0.8 \\
\hline & Agility Comp. (s) & 89 & $19.63^{*}$ & 0.08 & 38 & 20.02 & 0.15 & 0.37 & Small & $0.36^{\wedge}$ & 0.08 & $0.59^{\wedge}$ & 0.06 \\
\hline & 10 m Sprint (s) & 89 & $1.81^{*}$ & 0.01 & 37 & 1.86 & 0.02 & 0.45 & Medium & $0.01^{\wedge}$ & 0.00 & $0.01^{\wedge}$ & 0.00 \\
\hline & 30 m Sprint (s) & 89 & $4.55^{*}$ & 0.03 & 37 & 4.70 & 0.05 & 0.40 & Small & $0.06^{\wedge}$ & 0.01 & $0.03^{\wedge}$ & 0.00 \\
\hline & Yo-Yo IR1 (m) & 33 & 1325 & 52 & 17 & 1259 & 92 & -0.18 & Small & 38107 & 20290 & $99580^{\wedge}$ & 19584 \\
\hline \multirow[t]{9}{*}{ U15 } & CA (y) & 92 & 15.0 & 0.0 & 21 & 14.9 & 0.1 & -0.25 & Small & $0.0^{\wedge}$ & 0.0 & $0.1^{\wedge}$ & 0.0 \\
\hline & APHV (y) & 92 & 13.5 & 0.1 & 20 & 13.4 & 0.1 & -0.23 & Small & $0.2^{\wedge}$ & 0.0 & $0.0^{\wedge}$ & 0.0 \\
\hline & Height (cm) & 92 & 174.8 & 0.6 & 20 & 175.8 & 1.5 & 0.14 & Small & $33.4^{\wedge}$ & 4.6 & $2.2^{\wedge}$ & 0.2 \\
\hline & Body Mass (kg) & 92 & 64.6 & 0.8 & 20 & 68.0 & 1.8 & 0.36 & Small & $50.8^{\wedge}$ & 7.1 & $5.8^{\wedge}$ & 0.6 \\
\hline & $\mathrm{CMJ}(\mathrm{cm})$ & 91 & 37.5 & 0.5 & 20 & 35.2 & 1.3 & -0.37 & Small & $21.2^{\wedge}$ & 3.6 & $10.7^{\wedge}$ & 1.2 \\
\hline & Agility Comp. (s) & 92 & $19.11 *$ & 0.06 & 20 & 19.43 & 0.15 & 0.44 & Small & $0.28^{\wedge}$ & 0.05 & $0.14^{\wedge}$ & 0.01 \\
\hline & 10 m Sprint (s) & 92 & 1.76 & 0.01 & 20 & 1.78 & 0.02 & 0.31 & Small & $0.00^{\wedge}$ & 0.00 & $0.00^{\wedge}$ & 0.00 \\
\hline & 30 m Sprint (s) & 92 & 4.38 & 0.02 & 20 & 4.40 & 0.05 & 0.08 & Small & $0.03^{\wedge}$ & 0.01 & $0.02^{\wedge}$ & 0.00 \\
\hline & Yo-Yo IR1 (m) & 33 & 1563 & 53 & 10 & 1374 & 120 & -0.49 & Medium & 30648 & 23197 & 156153^ & 27631 \\
\hline \multirow[t]{8}{*}{ U16 } & CA (y) & 58 & $16.0^{*}$ & 0.0 & 48 & 15.8 & 0.1 & -0.60 & Medium & $0.0^{\wedge}$ & 0.0 & $0.1^{\wedge}$ & 0.0 \\
\hline & APHV (y) & 54 & 13.7 & 0.1 & 41 & 13.6 & 0.1 & -0.04 & Small & $0.3^{\wedge}$ & 0.0 & $0.0^{\wedge}$ & 0.0 \\
\hline & Height (cm) & 54 & 178.1 & 0.8 & 42 & 177.8 & 1.2 & -0.03 & Small & $33.2^{\wedge}$ & 4.9 & $1.3^{\wedge}$ & 0.2 \\
\hline & Body Mass (kg) & 54 & 69.4 & 0.9 & 42 & 68.4 & 1.4 & -0.11 & Small & $42.4^{\wedge}$ & 6.4 & $3.3^{\wedge}$ & 0.4 \\
\hline & $\mathrm{CMJ}(\mathrm{cm})$ & 53 & 37.9 & 0.6 & 38 & 38.0 & 1.0 & 0.02 & Small & $18.0^{\wedge}$ & 3.3 & $6.9^{\wedge}$ & 1.0 \\
\hline & Agility Comp. (s) & 52 & 18.80 & 0.07 & 41 & 18.86 & 0.11 & 0.08 & Small & $0.19^{\wedge}$ & 0.04 & $0.15^{\wedge}$ & 0.02 \\
\hline & 10 m Sprint (s) & 54 & $1.74 *$ & 0.01 & 42 & 1.77 & 0.01 & 0.37 & Small & $0.00^{\wedge}$ & 0.00 & $0.00^{\wedge}$ & 0.00 \\
\hline & 30 m Sprint (s) & 54 & 4.27 & 0.03 & 42 & 4.35 & 0.04 & 0.29 & Small & $0.03^{\wedge}$ & 0.01 & $0.01^{\wedge}$ & 0.00 \\
\hline
\end{tabular}




\begin{tabular}{|c|c|c|c|c|c|c|c|c|c|c|c|c|c|}
\hline & Yo-Yo IR2 (m) & 23 & 917 & 66 & 8 & 730 & 128 & -0.49 & Medium & $84788^{\wedge}$ & 25174 & $24122^{\wedge}$ & 5673 \\
\hline \multirow[t]{8}{*}{ U18 } & CA (y) & 60 & 17.3 & 0.1 & 29 & 17.4 & 0.1 & 0.10 & Small & $0.1^{\wedge}$ & 0.0 & $0.3^{\wedge}$ & 0.0 \\
\hline & Height (cm) & 58 & 180.9 & 0.8 & 29 & 180.4 & 1.4 & -0.04 & Small & $38.8^{\wedge}$ & 5.9 & $0.6^{\wedge}$ & 0.0 \\
\hline & Body Mass (kg) & 58 & 73.5 & 0.9 & 29 & 74.5 & 1.5 & 0.08 & Small & $43.5^{\wedge}$ & 6.8 & $4.0^{\wedge}$ & 0.3 \\
\hline & CMJ $(\mathrm{cm})$ & 60 & 40.1 & 0.5 & 28 & 39.1 & 0.9 & -0.14 & Small & $13.5^{\wedge}$ & 2.3 & $6.7 \wedge$ & 0.6 \\
\hline & Agility Comp. (s) & 60 & 18.55 & 0.06 & 27 & 18.68 & 0.11 & 0.17 & Small & $0.17^{\wedge}$ & 0.03 & $0.13^{\wedge}$ & 0.01 \\
\hline & 10 m Sprint (s) & 60 & 1.72 & 0.01 & 28 & 1.75 & 0.02 & 0.21 & Small & $0.00^{\wedge}$ & 0.00 & $0.00^{\wedge}$ & 0.00 \\
\hline & 30 m Sprint (s) & 60 & $4.20 *$ & 0.02 & 28 & 4.28 & 0.04 & 0.26 & Small & $0.03^{\wedge}$ & 0.01 & $0.01^{\wedge}$ & 0.00 \\
\hline & Yo-Yo IR2 (m) & 26 & 820 & 37 & 12 & 877 & 66 & 0.21 & Small & $18445^{\wedge}$ & 8265 & $43109 \wedge$ & 6916 \\
\hline \multirow[t]{8}{*}{ U21 } & CA (y) & 34 & 19.0 & 0.1 & 37 & 19.1 & 0.2 & 0.03 & Small & $0.3^{\wedge}$ & 0.1 & $0.4 \wedge$ & 0.0 \\
\hline & Height (cm) & 31 & 183.3 & 1.1 & 25 & 181.9 & 1.6 & -0.14 & Small & $37.0^{\wedge}$ & 7.0 & $0.3^{\wedge}$ & 0.0 \\
\hline & Body Mass (kg) & 33 & 78.1 & 1.2 & 35 & 76.7 & 1.6 & -0.11 & Small & $43.6^{\wedge}$ & 7.7 & $4.1^{\wedge}$ & 0.4 \\
\hline & $\mathrm{CMJ}(\mathrm{cm})$ & 32 & 40.5 & 0.9 & 34 & 40.2 & 1.2 & -0.03 & Small & $19.2^{\wedge}$ & 4.2 & $9.7 \wedge$ & 1.3 \\
\hline & Agility Comp. (s) & 32 & $18.22 *$ & 0.09 & 34 & 18.48 & 0.12 & 0.29 & Small & $0.20^{\wedge}$ & 0.04 & $0.09^{\wedge}$ & 0.01 \\
\hline & 10 m Sprint (s) & 32 & 1.69 & 0.01 & 35 & 1.71 & 0.02 & 0.14 & Small & $0.00^{\wedge}$ & 0.00 & $0.00^{\wedge}$ & 0.00 \\
\hline & 30 m Sprint (s) & 32 & 4.11 & 0.03 & 35 & 4.14 & 0.04 & 0.10 & Small & $0.02^{\wedge}$ & 0.01 & $0.01^{\wedge}$ & 0.00 \\
\hline & Yo-Yo IR2 (m) & 20 & 880 & 45 & 13 & 924 & 74 & 0.14 & Small & 14411 & 10309 & $70397 \wedge$ & 12143 \\
\hline
\end{tabular}

Notes: $\mathrm{n}$ = total number of individual players observed; $\mathrm{ES}=$ effect size; $\mathrm{SE}=$ Standard Error; $\mathrm{CA}=$ chronological age; APHV = age at peak height velocity; CMJ = countermovement jump; Agility Comp. = agility composite; Yo-Yo IR1 or IR2 = Yo-Yo Intermittent Recovery Test Level 1 or 2. Values are provided as means and SE.

* denotes significant difference $(\mathrm{P}<0.05)$ compared to Dropout; ^ denotes significant $(\mathrm{P}<0.05)$ between or within-subject variance 
Table 3 Proportions (in \%) of elite youth players that were retained into the subsequent age group between 2010/11 and 2016/17 seasons.

\begin{tabular}{|c|c|c|c|c|c|c|c|c|c|c|c|}
\hline & \multicolumn{11}{|c|}{ Transition to age group } \\
\hline & U12 & U13 & U14 & U15 & U16 & U17 & U18 & U19 & U20 & U21 & First Team \\
\hline U11 & 84.3 & 60.9 & 47.8 & 28.7 & 18.3 & 8.7 & 5.2 & 1.7 & - & - & - \\
\hline U12 & & 97.3 & 78.4 & 40.5 & 27.0 & 13.5 & 10.8 & 2.7 & - & - & - \\
\hline U13 & & & 97.0 & 48.5 & 33.3 & 33.3 & 12.1 & 9.1 & 6.1 & - & - \\
\hline U14 & & & & 73.0 & 51.4 & 51.4 & 8.1 & 5.4 & 2.7 & 2.7 & 2.7 \\
\hline U15 & & & & & 78.9 & 28.9 & 23.7 & 10.5 & 7.9 & 2.6 & - \\
\hline U16 & & & & & & 88.0 & 76.0 & 40.0 & 24.0 & 20.0 & 8.0 \\
\hline U17 & & & & & & & 89.7 & 55.2 & 37.9 & 17.2 & - \\
\hline U18 & & & & & & & & 85.7 & 57.1 & 35.7 & 21.4 \\
\hline U19 & & & & & & & & & 87.0 & 56.5 & 30.4 \\
\hline U20 & & & & & & & & & & 50.0 & 25.0 \\
\hline
\end{tabular}

Note: Percentages should be interpreted row-wise and refer to all players having played in the previous age group (column). 


\section{Discussion}

The purpose of this study was to longitudinally investigate factors associated with player retention from each age group within an elite football academy. Specifically, we examined whether birth quartile, somatic maturity, anthropometry, and physical performance characteristics were able to distinguish retained and dropout players between $\mathrm{U} 11$ to $\mathrm{U} 21$ groups. This is the first study to investigate player retention across the developmental pathway within an English Category 1 academy, which includes the use of multilevel modelling for statistical analysis.

Our findings for the first aim of this study revealed that despite an overrepresentation of relatively older players, once selected into this academy, birth quartile did not exert a significant influence on the likelihood of being retained throughout the developmental pathway. To the authors' awareness, the relationship between birth date and retention/dropout, after selection into an elite youth academy, has seldom been addressed within previous research ${ }^{9,34}$. Still, our results corroborate the findings within a Spanish football academy, in which birth date was not associated with being retained or promoted to a higher playing level between U14 and U18 groups ${ }^{35}$. However, it must be acknowledged that the low number of Q3 and Q4 players within the current study represent drawbacks in the analysis. Indeed, future studies would benefit from adopting a longer study period and/or a larger sample size in order to improve statistical power, and thus yield stronger inferences.

Recent research conducted within football and ice hockey demonstrates that dropout is enhanced for Q4 players between $10-13$ and $10-15$ years of age, respectively ${ }^{36,37}$. Our findings within U11 and U12 groups (Table 1), demonstrate that Q4 have a lower (OR: 0.4) and similar (OR: 1.0) likelihood of being retained compared to Q1 players, respectively. Although, it must be noted that the total number of Q4 players was low in comparison with other quartiles for each age group. A number of factors have been identified to contribute to dropout in youth sport, with a systematic review conducted by Crane and Temple (2015) reporting that perceptions of physical or sport competence, as well as relative age are associated ${ }^{38}$. In support of these findings, Figueiredo et al. observed that the enhanced dropout of Q4 players may be related to coaches' perceptions of talent/competence; Q1 players were rated higher, had more entries to elite teams and less dropouts in subsequent years compared to Q4 players ${ }^{37}$. Additionally, the authors observed that despite being advanced in maturity, Q4 players typically demonstrated lower actual competence (assessed via physical performances and sport-specific skills) when compared to other birth quartiles. Therefore, it appears that Q4 players not only face discrimination that denies them selection into academies at a young age ${ }^{34}$, but also from progressing from the youngest age groups within them, which may be related to perceptions of and/or actual competence. However, it was 
beyond the scope of this study to examine the coaches' perceptions of player competence in relation to retention/dropout, yet this warrants further exploration.

Similarly, Helsen et al. observed that football players born later in the selection year tended to dropout from 12 years of age ${ }^{39}$. Yet, within this academy, Q4 contributed a low number of players to the overall dropout from each age group, and typically had a slightly higher likelihood of being retained ( $\geq 1.4$ times) in comparison with Q1, between U13 and U16 groups (Table 1). This suggests that the small number of relatively younger players selected into this academy are considered to be highly talented, thereby facilitating their progression along the developmental pathway. On the other hand, there was a large number of Q1 players that dropout from this academy suggesting that many of these are erroneously recruited at a young age due to the relative age selection bias, which is robust within English youth football ${ }^{15,} 40-42$. Moreover, given that the prevalence of the RAE throughout this particular academy is strong ${ }^{41}$, it seems that the Q1 dropouts are typically being replaced by relatively older players (i.e. high turnover of Q1 players), which supports the findings of Bidaurrazaga-Letona et al. ${ }^{43}$. Consequently, many talented relatively younger players may miss developmental opportunities associated with systematic training and competition within academies ${ }^{44,45}$, and thus dropout from football (at all playing levels) prematurely ${ }^{39}$.

In the U18 group, Q4 had a similar likelihood of retention compared to Q1 (Table 1), though a large difference was observed in the U21 group, with Q4 players demonstrating an enhanced likelihood of being retained. The only statistically significant difference was observed in the U21 group, where Q3 players had a greater likelihood of being retained compared to Q1 players, though it is plausible that this is result of a multiple comparisons issue. Nevertheless, such findings may indicate that at the end of adolescence, the small number of relatively younger players that have persisted demonstrate superior competencies. Actually, allowing for the lower overall number of Q4 players in the U21 group, their higher proportion of retention compared to other birth quartiles aligns with previous findings in German football ${ }^{20,46}$ and English rugby union ${ }^{47}$, highlighting potential long-term advantages for relatively younger players.

Taken together, our results demonstrating a typically higher retention of Q4 players throughout the developmental pathway may be attributed to advanced maturity of Q4 players ${ }^{14,48}$ and/or the 'underdog' hypothesis ${ }^{22}$. The latter intimates that developmental advantages are gained through competing with relatively older and/or earlier maturing peers, which may include psychological effects ${ }^{47}$ and compensation in technical skills ${ }^{49}$. In contrast, more recent evidence indicates that relatively older players within Australian football have long-term career advantages, which may be attributed to developmental opportunities gained from enhanced exposure from a young age ${ }^{50}$. Unfortunately, it was beyond the scope of this study to investigate this notion further, though future research that examines developmental opportunities for both relatively older and younger players in a longitudinal manner would be useful. 
Closer inspection of the individuals that were retained from the U21 group revealed that of the three Q4 players, only one was present at U18 - the point which he was also signed. Thus, the limited number of Q4 players that were retained at U21 were recruited towards the end of adolescence from clubs competing in lower divisions. On the other hand, six of the ten Q1 players that were retained at U21 were registered to the club by U16 (or below), demonstrating that they persisted longer within this top categorised academy. This finding could imply different routes to attaining professional status for relatively older and younger players, which may relate to individualistic and collectivistic approaches ${ }^{51}$, respectively, though further research is required. Still, the greater overall number of Q1 and Q2 players being retained from the U21 group (in comparison with Q4 players) is consistent with evidence demonstrating relative age effects persist into the top professional leagues in England ${ }^{52,53}$.

Other key findings of this study relate to aspects of the player retention process throughout the developmental pathway. Table 1 indicates a high percentage (i.e. $>84 \%$ ) of players retained from U11 to U13 groups in comparison with the U14 group (66.2\%). Subsequently, the proportion of retained players typically decreases progressively from the U15 group ( $81.6 \%$ ) to the U21 group (45.1\%), with the second lowest proportion of retention within this academy observed in the U16 group (54.1\%). These findings lack concordance with elite youth teams from Belgium, where the percentage of retained players remained consistent (i.e. $73-78 \%$ ) between $U 11$ and $U 16$ groups ${ }^{25}$. This could suggest that talent identification within this academy prior to U14 and U16 groups is somewhat ineffective given the high turnover of players from these groups.

Alternatively, it may reflect the financial constraints associated with adopting a full time training model and/or providing scholarships ${ }^{1}$, thereby limiting the number of players that can be retained from these groups. The supplementary data presented in Table 3 shows the proportion of players that are retained from each age group and subsequently progress along the developmental pathway. Importantly, less than $50 \%$ of players were retained for at least three seasons (except for players recruited at U14) and less than $13 \%$ of players from each age group between U11 to U14 progress to the U18 group within this academy. The low proportion of retention along the developmental pathway corresponds with observations from German academy and youth national teams - implying a collectivistic approach for players that eventually attain professional and national team status ${ }^{51,54}$. These findings highlight that policymakers in youth football should be cognisant of individualistic and collectivistic approaches, and the potential for the former - which is currently emphasised in English academies $^{1}$ - to exert an unintended effect on the latter within lower categorised academies and/or grassroots clubs ${ }^{51}$.

The second aim of this study was to investigate the differences in somatic maturity, anthropometric and physical performance characteristics between retained and dropout players from U11 to U21 groups. Multilevel modelling revealed significant differences amongst these factors in an age group dependent manner (Table 2). This contrasts previous research demonstrating the unsuitability of these factors in 
distinguishing the most talented players within highly selective cohorts and exposed to systematic training ${ }^{23,24}$. Specifically, our results demonstrate that between U11 to U21 groups, retained players were typically superior in physical performance tests, where effect sizes were typically small to medium (Table 2). This corroborates with previous findings that retained players within European football academies $25,35,43$ and Australian football ${ }^{55}$ achieved superior physical performances. Buchheit et al. reported that performances on field tests were related to match-running performance (in a positiondependent manner) ${ }^{56}$, where superior match-running performance is also related to retention across age groups within an English academy ${ }^{57}$. Of note, our findings also revealed that agility T-test performance was superior for retained players in five out of the eight age groups investigated. This suggests that the agility T-test is particularly valuable for distinguishing players that progress along the developmental pathway within a highly selective cohort. These findings highlight the influence of physical performances on the selection process that operates within youth football academies.

In contrast to the findings by Deprez et al., we did not observe any clear trends for physical performances to distinguish retained players in relation to the timing of peak height velocity ${ }^{25}$. Although, our findings for the U14 group appear pertinent in that agility T-test and sprint performances were all significantly discriminant, suggesting that retained players experienced performance enhancements concomitant with peak height velocity ${ }^{58}$. The discrepancy between both studies may reflect differences in the samples investigated and/or the importance of specific factors that influence retention within high-level youth teams across countries, though this warrants further investigation.

The second aim also revealed that retained players were typically advanced in maturity (i.e. lower APHV), taller, and heavier than dropouts (Table 2), with a greater number of significant differences for these variables evident in comparison with Deprez et al. ${ }^{25}$. Specifically, as significant differences for these variables were evident in U13 and U14 groups (especially the latter), which aligns with mean values for estimated APHV, it suggests that retained players were experiencing accelerated growth. Comparisons with UK normative height values indicates that retained players between U11 and U18 groups were typically just above the $75^{\text {th }}$ centile, whereas dropouts were typically just below the $75^{\text {th }}$ centile ${ }^{59}$. Interestingly, retained players in U13 and U14 groups were between the $75^{\text {th }}$ and $91^{\text {st }}$ centile, with dropouts residing around the $50^{\text {th }}$ and $75^{\text {th }}$ centile, respectively. This highlights that retention in these groups favours individuals undergoing an earlier onset and/or advanced tempo in growth - corresponding with research from Australian football 55 - where superior height may enhance a coach's perception of player giftedness ${ }^{60}$.

Within U18 and U21 groups, there was a tendency for retained players to demonstrate superior anthropometric and physical performance characteristics compared to dropouts, with significant differences observed for $30 \mathrm{~m}$ sprint and agility T-test, respectively (Table 2). This partially supports the findings by Emmonds et al., who observed that players offered professional contracts at U18 achieved superior sprint (10 
and $20 \mathrm{~m}$ ) and Yo-Yo IR2 performances ${ }^{61}$. Given that retained players were significantly younger in both U18 and U21 groups, we propose several explanations for our findings. First, this may simply reflect an artefact of the methodological approach adopted, whereby players categorised as dropout were typically in their final year within each age group (i.e. one or two years older). Alternatively, the tendency to retain younger players may be related to a greater (perceived) potential for long-term improvements by talent selectors. Finally, the lack of significant differences between retained and dropout players in these groups could suggest that, as both groups precede the First Team, other relevant predictors of performance could discriminate between the most talented players $2,35,43$. In any case, additional research in these age groups is warranted to better understand the factors that influence progression to the First Team squad.

A synthesis of analyses from both aims of this study revealed that U14 and U16 groups had an atypically low percentage of retention (Table 1), with retained players demonstrating superior age and sprint performance in both groups - similar to previous findings ${ }^{25,61}$, as well as superior body size and agility performance in the U14 group (Table 2). The emphasis on running-based performances for retention in both groups is likely related to the observation that match-running performance requirements (e.g. total and sprinting distance and peak game speed) typically increase in subsequent age groups following retention from U14 and U16 groups ${ }^{56,62}$. Furthermore, sprinting is an important action during decisive moments within football, such as reaching a ball before an opponent to score or prevent a goal ${ }^{63}$.

The findings from this study highlight that somatic maturity, anthropometric and physical performance characteristics exert an influence on the decision-making process regarding player retention within this elite academy. This contradicts data obtained from Australia in which youth coaches and recruitment staff indicated that technical, tactical and psychological attributes are the most important factors in terms of talent identification ${ }^{64}$. On the other hand, our findings appear to align with research establishing that organisational pressures and physical maturity exert an influence on the selection process adopted by the coaches of 12 to 15 -year-old football players ${ }^{65}$. However, whilst there is some indication, albeit limited, that physical performances are related to future career success ${ }^{4,66}$, the long-term stability of physical characteristics is questionable, thereby restricting their suitability for talent identification and selection processes within youth football ${ }^{67}$. Especially as recent studies in youth football players demonstrate that peak development of the discriminating factors we observed between U11 to U14 groups (i.e. anthropometry and physical performances) do not subside until around 15-17 years of age or post-PHV ${ }^{68,69}$. Therefore, in order for clubs to recruit and develop the most talented players, decision-making processes regarding player retention would benefit from placing less emphasis on anthropometric and physical performance characteristics until this time ${ }^{55}$.

We do acknowledge several limitations of this study. First, the low number of Q4 players we observed for this study is typical of football academies, where previous research has 
accounted for this by using bi-annual age groups for statistical analysis ${ }^{15}$. However, this may mask important information that operates in an age group dependent manner. Additionally, it must be noted that retention/dropout may be influenced by the age at which the individual first joined the academy ${ }^{4}$ (among other factors), and thus future studies should seek to account for this. Thus, we suggest the findings for aim one of this study be interpreted with caution. Secondly, this study reports the findings from one professional football club in England, and therefore the generalisability of findings is unclear. Furthermore, this study did not measure any other qualities that are deemed relevant for football performance ${ }^{2}$ and thus retention/dropout. Issues regarding the assessment of somatic maturity have been discussed elsewhere ${ }^{70,71}$, though we considered the current method ${ }^{30}$ to be the most viable option for the purposes of this study. Finally, it is acknowledged that the raw dataset used in this study (i.e. without ascribing retained and dropout groups), which has been obtained from an applied setting, was also available to the coaches at the club, and thus may have influenced the decision-making process on player retention. However, it was beyond the scope of this study to determine if this was the case in the current club, though other research indicates that knowledge of birth date does not nullify the relative age selection bias ${ }^{65}$. Still, qualitative research is warranted to gain a clearer understanding of the decisionmaking process adopted by coaches and other talent selectors; specifically, the identification of multidisciplinary factors that influence selection and retention in highly selective cohorts would be useful.

In terms of future directions, the implementation of suggested approaches such as a 'Futures' team ${ }^{72}$ and/or 'bio-banding' 73 may enable talented youth players demonstrating inferior maturity and/or physical attributes an opportunity to continue development in an elite environment as opposed to premature dropout. With regards to the latter, this has recently been investigated from a player development perspective, where both early and late maturing players reported positive experiences ${ }^{74}$. This observation would appear to have implications for early maturers in particular, given that the additional challenge provided by bio-banding may facilitate greater developmental opportunities for these players ${ }^{75}$. In any case, there is currently a lack of empirical evidence justifying the use of these approaches for player selection and retention purposes. Additionally, future research identifying multidisciplinary factors that influence retention within highly selective teams would be useful, which could include longitudinal investigations of motor coordination 25,72 as well as technical, tactical, psychological, and sociological factors ${ }^{2}$. Indeed, several other authors have reported that multidisciplinary factors are able to distinguish the most successful youth players $4,5,7,49,76$. Similarly, additional research that considers the most pertinent factors associated with dropout is required, particularly within the unique context of football academies ${ }^{38}$. Such investigations would help to provide greater context to the observation that the standard errors for investigated variables in this study typically overlapped between retained and dropout players. This suggests that some dropouts may have demonstrated similar (or even superior) characteristics to retained players, 
yet likely had weaknesses for other competencies necessary for football performance, and thus warrants further investigation.

\section{Conclusion}

The first aim of this study revealed that birth quartile does not exert a significant influence on being retained within an English Category 1 academy, between U11 and U21 groups. Still, players born in Q4, albeit low in number, typically demonstrate an enhanced likelihood of persisting between U13 to U21 groups compared to Q1 players, and there is a seemingly high turnover of Q1 players throughout the developmental pathway. The second aim of this study revealed that retained players typically demonstrated superior age, somatic maturity, anthropometric and physical performance characteristics between U11 to U21 groups, with significant discriminatory factors observed in age group dependent manner and small to medium effect sizes typically observed. Taken together, our findings demonstrate that once selected into this English Category 1 academy, birth quartile does not significantly affect retention, yet somatic maturity, anthropometry and physical performance characteristics are able to distinguish individuals that are retained along the developmental pathway. Given the transient nature of these factors, youth football clubs should seek to place less emphasis on these during the selection and retention process of players. Instead, clubs should seek to adopt a multidisciplinary and dynamic approach that considers these alongside technical, tactical, psychological and sociological factors to prevent unnecessary discrimination and loss of talented young players.

\section{Acknowledgements}

We would like to thank everyone involved with this football club for supporting this study and providing the data. Furthermore, we want to express our gratitude to the reviewers for helping to improve this manuscript.

\section{Funding}

The author(s) received no financial support for the research, authorship, and/or publication of this article. 


\section{References:}

1. Premier League. Elite Player Performance Plan. Premier League; Gloucester Place, London, England.2011.

2. Williams AM and Reilly T. Talent identification and development in soccer. $J$ Sports Sci. 2000; 18: 657-67.

3. Figueiredo AJ, Gonçalves CE, Coelho e Silva MJ and Malina RM. Characteristics of youth soccer players who drop out, persist or move up. J Sports Sci. 2009; 27: 883-91.

4. Huijgen $\mathrm{BCH}$, Elferink-Gemser MT, Lemmink KAPM and Visscher C.

Multidimensional performance characteristics in selected and deselected talented soccer players. European Journal of Sport Science. 2014; 14: 2-10.

5. Vaeyens R, Malina RM, Janssens $M$, et al. A multidisciplinary selection model for youth soccer: the Ghent Youth Soccer Project. Br J Sports Med. 2006; 40: 928-34.

6. Toering T, Elferink-Gemser MT, Jordet G, Pepping GJ and Visscher C. Selfregulation of learning and performance level of elite youth soccer players. Int J Sport Psychol. 2012; 43: 312-25.

7. Reilly $T$, Williams AM, Nevill A and Franks A. A multidisciplinary approach to talent identification in soccer. J Sports Sci. 2000; 18: 695-702.

8. Coelho ESMJ, Figueiredo AJ, Simoes F, et al. Discrimination of u-14 soccer players by level and position. Int J Sports Med. 2010; 31: 790-6.

9. Sarmento H, Anguera MT, Pereira A and Araujo D. Talent Identification and Development in Male Football: A Systematic Review. Sports Med. 2018; 48: 907-31.

10. Mujika I, Vaeyens R, Matthys SPJ, Santisteban J, Goiriena J and Philippaerts R. The relative age effect in a professional football club setting. J Sports Sci. 2009; 27: 1153-8.

11. Musch J and Grondin S. Unequal competition as an impediment to personal development: A review of the relative age effect in sport. Dev Rev. 2001; 21: 147-67.

12. Carling C, Le Gall F and Malina RM. Body size, skeletal maturity, and functional characteristics of elite academy soccer players on entry between 1992 and 2003. J Sports Sci. 2012; 30: 1683-93.

13. Malina RM, Chamorro M, Serratosa L and Morate F. TW3 and Fels skeletal ages in elite youth soccer players. Ann Hum Biol. 2007; 34: 265-72.

14. Deprez D, Coutts AJ, Fransen J, et al. Relative Age, Biological Maturation and Anaerobic Characteristics in Elite Youth Soccer Players. Int J Sports Med. 2013; 34: 897903.

15. Lovell R, Towlson C, Parkin G, Portas M, Vaeyens R and Cobley S. Soccer Player Characteristics in English Lower-League Development Programmes: The Relationships 
between Relative Age, Maturation, Anthropometry and Physical Fitness. PLoS One. 2015; 10: e0137238.

16. Cobley S, Baker J, Wattie $\mathrm{N}$ and McKenna J. Annual age-grouping and athlete development. Sports Med. 2009; 39: 235-56.

17. Figueiredo AJ, Coelho e Silva MJ and Malina RM. Predictors of functional capacity and skill in youth soccer players. Scand J Med Sci Sports. 2011; 21: 446-54.

18. Delorme N, Boiche J and Raspaud M. Relative age and dropout in French male soccer. J Sports Sci. 2010; 28: 717-22.

19. Malina RM, Pena Reyes ME, Eisenmann JC, Horta L, Rodrigues J and Miller R. Height, mass and skeletal maturity of elite Portuguese soccer players aged 11-16 years. $J$ Sports Sci. 2000; 18: 685-93.

20. Skorski S, Skorski S, Faude O, Hammes D and Meyer T. The Relative Age Effect in German Elite Youth Soccer: Implications for a Successful Career. Int J Sports Physiol Perform. 2016; 11: 370-6.

21. Ostojic SM, Castagna C, Calleja-Gonzalez J, Jukic I, Idrizovic K and Stojanovic M. The biological age of 14-year-old boys and success in adult soccer: do early maturers predominate in the top-level game? Res Sports Med. 2014; 22: 398-407.

22. Gibbs BG, Jarvis JA and Dufur MJ. The rise of the underdog? The relative age effect reversal among Canadian-born NHL hockey players: A reply to Nolan and Howell. International Review for the Sociology of Sport. 2012; 47: 644-9.

23. le Gall F, Carling C, Williams M and Reilly T. Anthropometric and fitness characteristics of international, professional and amateur male graduate soccer players from an elite youth academy. J Sci Med Sport. 2010; 13: 90-5.

24. Franks A, Williams, AM, Reilly, T. \& Nevill, A. Talent identification in elite youth soccer players: Physical and physiological characteristics. Communication to the 4th World Congress on Science and Football, Sydney. J Sports Sci. 1999; 17: 812.

25. Deprez DN, Fransen J, Lenoir M, Philippaerts RM and Vaeyens R. A retrospective study on anthropometrical, physical fitness, and motor coordination characteristics that influence dropout, contract status, and first-team playing time in high-level soccer players aged eight to eighteen years. J Strength Cond Res. 2015; 29: 1692-704.

26. Stewart A, Marfell-Jones M, Olds T and de Ridder H. International standards for anthropometric assessment ISAK: The International Society for the Advancement of Kinanthropometry. Lower Hutt, New Zealand: ISAK, 2011.

27. Semenick D. Tests and Measurements: The T-test. Strength \& Conditioning Journal. 1990; 12: 36-7.

28. Krustrup $\mathrm{P}, \mathrm{Mohr} \mathrm{M}$, Amstrup $\mathrm{T}$, et al. The yo-yo intermittent recovery test: physiological response, reliability, and validity. Med Sci Sports Exerc. 2003; 35: 697-705. 
29. Krustrup P, Mohr M, Nybo L, Jensen JM, Nielsen JJ and Bangsbo J. The Yo-Yo IR2 test: physiological response, reliability, and application to elite soccer. Med Sci Sports Exerc. 2006; 38: 1666-73.

30. Mirwald RL, Baxter-Jones AD, Bailey DA and Beunen GP. An assessment of maturity from anthropometric measurements. Med Sci Sports Exerc. 2002; 34: 689-94.

31. Goldstein H. Multilevel Statistical Models. 2nd ed. London, Edward Arnold; New York, Halstead Press.

32. Charlton C, Rasbash, J, Browne, WJ, Healy, M and Cameron, B. (2019) MLwiN Version 3.03. Centre for Multilevel Modelling, University of Bristol.

33. Cohen J. Statistical power analysis for the behavioral sciences. Hillsdale, N.J.: L. Erlbaum Associates, 1988.

34. Sierra-Diaz MJ, Gonzalez-Villora S, Pastor-Vicedo JC and Serra-Olivares J. Soccer and Relative Age Effect: A Walk among Elite Players and Young Players. Sports (Basel, Switzerland). 2017; 5: pii: E5.

35. Castillo D, Perez-Gonzalez B, Raya-Gonzalez J, Fernandez-Luna A, Burillo P and Lago-Rodriguez A. Selection and promotion processes are not associated by the relative age effect in an elite Spanish soccer academy. PLoS One. 2019; 14: e0219945.

36. Lemez S, Baker J, Horton S, Wattie $\mathrm{N}$ and Weir P. Examining the relationship between relative age, competition level, and dropout rates in male youth ice-hockey players. Scand J Med Sci Sports. 2014; 24: 935-42.

37. Figueiredo AJ, Coelho ESMJ, Cumming SP and Malina RM. Relative age effect: Characteristics of youth soccer players by birth quarter and subsequent playing status. $J$ Sports Sci. 2018; 37: 677-84.

38. Crane J and Temple V. A systematic review of dropout from organized sport among children and youth. European Physical Education Review. 2015; 21: 114-31.

39. Helsen WF, Starkes JL and Van Winckel J. The influence of relative age on success and dropout in male soccer players. Am J Hum Biol. 1998; 10: 791-8.

40. Hill M, Scott S, Malina RM, McGee D and Cumming SP. Relative age and maturation selection biases in academy football. J Sports Sci. 2019: 1-9.

41. Patel R, Nevill A, Cloak R, Smith T and Wyon M. Relative age, maturation, anthropometry and physical performance characteristics of players within an Elite Youth Football Academy. International Journal of Sports Science \& Coaching. 2019; 0: 1747954119879348.

42. Simmons $\mathrm{C}$ and Paull GC. Season-of-birth bias in association football. J Sports Sci. 2001; 19: 677-86.

43. Bidaurrazaga-Letona I, Lekue JA, Amado M and Gil SM. Progression in Youth Soccer: Selection and Identification in Youth Soccer Players Aged 13-15 Years. J Strength Cond Res. 2019; 33: 2548-58. 
44. Wrigley RD, Drust B, Stratton G, Atkinson G and Gregson W. Long-term soccerspecific training enhances the rate of physical development of academy soccer players independent of maturation status. Int J Sports Med. 2014; 35: 1090-4.

45. Valente-dos-Santos J, Coelho-e-Silva MJ, Simoes F, et al. Modeling developmental changes in functional capacities and soccer-specific skills in male players aged 11-17 years. Pediatr Exerc Sci. 2012; 24: 603-21.

46. Grossmann B and Lames M. Relative age effect (RAE) in football talents - The role of youth academies in transition to professional status in Germany. International Journal of Performance Analysis in Sport. 2013; 13: 120-34.

47. McCarthy $\mathrm{N}$ and Collins $\mathrm{D}$. Initial identification \& selection bias versus the eventual confirmation of talent: evidence for the benefits of a rocky road? J Sports Sci. 2014; 32: 1604-10.

48. Hirose N. Relationships among birth-month distribution, skeletal age and anthropometric characteristics in adolescent elite soccer players. J Sports Sci. 2009; 27: 1159-66.

49. Zuber C, Zibung M and Conzelmann A. Holistic Patterns as an Instrument for Predicting the Performance of Promising Young Soccer Players - A 3-Years Longitudinal Study. Front Psychol. 2016; 7: 1088.

50. Tribolet R, Watsford ML, Coutts AJ, Smith C and Fransen J. From entry to elite: The relative age effect in the Australian football talent pathway. J Sci Med Sport. 2019; 22: 741-5.

51. Güllich A. Selection, de-selection and progression in German football talent promotion. European Journal of Sport Science. 2014; 14: 530-7.

52. Fleming $\mathrm{J}$ and Fleming S. Relative age effect amongst footballers in the English Premier League and English Football League, 2010-2011. International Journal of Performance Analysis in Sport. 2012; 12: 361-72.

53. Rada A, Padulo J, Jelaska I, Ardigo LP and Fumarco L. Relative age effect and second-tiers: No second chance for later-born players. PLoS One. 2018; 13: e0201795.

54. Schroepf B and Lames M. Career patterns in German football youth national teams - A longitudinal study. International Journal of Sports Science \& Coaching. 2018; 13: 405-14.

55. Tribolet R, Bennett KJM, Watsford ML and Fransen J. A multidimensional approach to talent identification and selection in high-level youth Australian Football players. J Sports Sci. 2018; 36: 2537-43.

56. Buchheit M, Mendez-Villanueva A, Simpson BM and Bourdon PC. Match running performance and fitness in youth soccer. Int J Sports Med. 2010; 31: 818-25.

57. Goto H, Morris JG and Nevill ME. Motion analysis of U11 to U16 elite English Premier League Academy players. J Sports Sci. 2015; 33: 1248-58. 
58. Philippaerts $\mathrm{RM}$, Vaeyens $\mathrm{R}$, Janssens $\mathrm{M}$, et al. The relationship between peak height velocity and physical performance in youth soccer players. J Sports Sci. 2006; 24: 221-30.

59. Royal College of Paediatrics and Child Health. UK-WHO growth charts - 2-18 years. 2012.

60. Furley P and Memmert D. Coaches' implicit associations between size and giftedness: implications for the relative age effect. J Sports Sci. 2015: 1-8.

61. Emmonds S, Till K, Jones B, Mellis M and Pears M. Anthropometric, speed and endurance characteristics of English academy soccer players: Do they influence obtaining a professional contract at 18 years of age? International Journal of Sports Science \& Coaching. 2016; 11: 212-8.

62. Saward C, Morris JG, Nevill ME, Nevill AM and Sunderland C. Longitudinal development of match-running performance in elite male youth soccer players. Scand J Med Sci Sports. 2016; 26: 933-42.

63. Faude $\mathrm{O}$, Koch $\mathrm{T}$ and Meyer T. Straight sprinting is the most frequent action in goal situations in professional football. J Sports Sci. 2012; 30: 625-31.

64. Larkin $\mathrm{P}$ and $\mathrm{O}^{\prime}$ Connor D. Talent identification and recruitment in youth soccer: Recruiter's perceptions of the key attributes for player recruitment. PLoS One. 2017; 12: e0175716.

65. Hill B and Sotiriadou P. Coach decision-making and the relative age effect on talent selection in football. European Sport Management Quarterly. 2016; 16: 292-315.

66. Gonaus $\mathrm{C}$ and Muller E. Using physiological data to predict future career progression in 14- to 17-year-old Austrian soccer academy players. J Sports Sci. 2012; 30: 1673-82.

67. Buchheit M and Mendez-Villanueva A. Reliability and stability of anthropometric and performance measures in highly-trained young soccer players: effect of age and maturation. J Sports Sci. 2013; 31: 1332-43.

68. Fransen J, Bennett KJM, Woods CT, et al. Modelling age-related changes in motor competence and physical fitness in high-level youth soccer players: implications for talent identification and development. Science and Medicine in Football. 2017; 1: 203-8.

69. Towlson C, Cobley S, Parkin G and Lovell R. When does the influence of maturation on anthropometric and physical fitness characteristics increase and subside? Scand J Med Sci Sports. 2018; 28: 1946-55.

70. Lloyd RS, Oliver JL, Faigenbaum AD, Myer GD and De Ste Croix MBA. Chronological Age vs. Biological Maturation: Implications for Exercise Programming in Youth. J Strength Cond Res. 2014; 28: 1454-64. 
71. Mills K, Baker D, Pacey V, Wollin M and Drew MK. What is the most accurate and reliable methodological approach for predicting peak height velocity in adolescents? A systematic review. J Sci Med Sport. 2017; 20: 572-7.

72. Vandendriessche JB, Vaeyens R, Vandorpe B, Lenoir M, Lefevre J and Philippaerts RM. Biological maturation, morphology, fitness, and motor coordination as part of a selection strategy in the search for international youth soccer players (age 15-16 years). J Sports Sci. 2012; 30: 1695-703.

73. Cumming SP, Lloyd RS, Oliver JL, Eisenmann JC and Malina RM. Bio-banding in Sport: Applications to Competition, Talent Identification, and Strength and Conditioning of Youth Athletes. Strength \& Conditioning Journal. 2017; 39: 34-47.

74. Cumming SP, Brown DJ, Mitchell S, et al. Premier League academy soccer players' experiences of competing in a tournament bio-banded for biological maturation. J Sports Sci. 2018; 36: 757-65.

75. Collins $D$ and MacNamara A. The rocky road to the top: why talent needs trauma. Sports Med. 2012; 42: 907-14.

76. Forsman $\mathrm{H}$, Blomqvist $\mathrm{M}$, Davids $\mathrm{K}$, Liukkonen J and Konttinen N. Identifying technical, physiological, tactical and psychological characteristics that contribute to career progression in soccer. International Journal of Sports Science \& Coaching. 2016; 11: 505-13. 